\title{
Microwave-assisted rapid synthesis of alumina nanoparticles using tea, coffee and triphala extracts
}

\author{
Prasant Sutradhar • Narayan Debnath • \\ Mitali Saha
}

Received: 13 August 2013/Accepted: 16 October 2013/Published online: 9 November 2013

(C) Shanghai University and Springer-Verlag Berlin Heidelberg 2013

\begin{abstract}
Alumina nanoparticles (AlNP) were synthesized from aluminium nitrate using extracts of tea, coffee and triphala - a well known herbal plant as well as a nontoxic and eco-friendly green material. The synthesis was carried out taking 1:4 ratio of metal salt and these extracts under microwave irradiations at $540 \mathrm{~W}$, which gave better yield of nanoparticles. Water was taken as solvent medium. The formations of AlNP were initially monitored by the colour changes occurring in the reaction mixture during the incubation period. As synthesized nanoparticles were characterized by scanning electron microscope (SEM), UV-Visible (UV-Vis) spectroscopy and Fourier transform infrared spectroscopy (FTIR). The AINP were found to be spherical in shape in case of tea and coffee extracts with a size of 50-200 nm and to be oval shaped in case of triphala extract with an average size of 200-400 nm. The formation of AlNP with the microwave-assistance using these plant extracts has proved to be very faster than any other methods. In addition, excellent reproducibility of these nanoparticles, without the use of any additional capping agent or stabilizer will have great advantages in comparison with microbial synthesis, avoiding all the tedious and hygienic complications.
\end{abstract}

Keywords Alumina nanoparticles - Microwave · Tea $\cdot$ Coffee $\cdot$ Triphala

P. Sutradhar $\cdot$ N. Debnath $\cdot$ M. Saha $(\bowtie)$

Department of Chemistry, National Institute of Technology,

Agartala 799055, Tripura, India

e-mail: mitalichem71@gmail.com

\section{Introduction}

Nanocrystalline metal oxides are found to have immense applications in the fields of catalysts, semiconductors, medical science, capacitors, batteries, absorbents, chemical and biological sensors, optoelectronics, information storage photonic, electronic devices and in preparation of polymer nanocomposites with improved mechanical properties [1-4]. Various methods, such as wet chemical reduction, reverse micelles, electrochemical and nonelectrochemical techniques, microwave assisted process and nowdays via green chemistry route [5-9] have been developed to synthesize metal nanoparticles because of the diversity and importance of these applications. Use of plant sources or extracts in synthesis of nanoparticles is quite novel leading to truly green chemistry, which provides advancement over chemical and physical methods [10-12]. It is costly effective, environment friendly and can be easily scaled up for large scale synthesis and most importantly, there is no need in this method to use high pressure, energy, temperature and toxic chemicals. Biosynthesis of metal nanoparticles extracted from different parts of the plant is the most effective process of synthesis at a very affordable cost.

According to the researchers, the polyol components present in the plant extract are responsible for the reduction of metal ions, whereas water soluble heterocyclic components stabilize the formed nanoparticles. The synthesis of metal oxide nanoparticles by reduction of the corresponding metal salts via green chemistry mainly depends upon the choice of solvent, reducing agent employed and the capping agent. Silver, gold, platinum and palladium have been rottenly used for the synthesis of nanoparticles [13-16]. In general, alumina has many interesting properties such as catalyst, as high stability and hardness, 
insulation, surface protective coatings, as composite materials with tuneable mechanical properties, etc [4, 17]. In the recent years, AlNP were synthesized in liquid using a short pulse laser with the pulse width in the range of nanosecond [18]. In continuation of our earlier studies to synthesize metal nanoparticles [19], we now have reported the microwave assisted green chemistry to synthesize AlNP using tea, coffee and triphala extracts. To the best of our knowledge, the preparation of AlNP in water using these extracts is so far unexplored under microwave irradiation. This approach has the basic advantage that no other stabilizing agent or capping agent is required to stabilize these nanoparticles.

\section{Materials and methods}

\subsection{Preparation of tea, coffee and triphala extracts}

Three plant extracts were used to produce AlNP. $10 \mathrm{~g}$ fine grounded powders of tea, coffee and crushed triphala were dissolved in $100 \mathrm{ml}$ water and boiled for around $2 \mathrm{~h}$. These were then centrifuged, filtered and stored in a cool place. After cooling at room temperature, these were centrifuged for $15 \mathrm{~min}$ and filtered. The filtrates were stored at $5-10{ }^{\circ} \mathrm{C}$ and used as reducing and stabilizing agents.

\subsection{Synthesis of AlNP using tea extract}

Aluminium nitrate was used as precursor for the synthesis of AlNP. To make sure a complete reduction, AlNP was dissolved in aqueous tea extract taking 1:4 ratio $(w / w)$ with stirring at room temperature. It was then subjected to microwave heating at $540 \mathrm{~W}$, which produced yellowish- brown precipitate after 6-7 min. The precipitates were centrifuged and washed with distilled water and methanol. These were further kept for drying at $100{ }^{\circ} \mathrm{C}$ for $3-4 \mathrm{~h}$.

\subsection{Synthesis of AlNP using coffee extract}

Aluminium nitrate and coffee extract taken in 1:4 ratio $(w / w)$ were subjected to microwave heating at $540 \mathrm{~W}$ in a similar way mentioned above. After 5-6 min, yellowish-brown precipitate was observed. The precipitate was centrifuged, washed with distilled water and methanol, and then kept for drying at $100{ }^{\circ} \mathrm{C}$ for $3-4 \mathrm{~h}$.

\subsection{Synthesis of AlNP using triphala extract}

Aluminium nitrate was added in triphala extract 1:4 ratio $(w / w)$, and the solution was subjected to microwave heating at $540 \mathrm{~W}$. Within 2-3 min light brown coloured precipitate was formed. The precipitate was centrifuged, washed with distilled water, and methanol and kept for drying at $100{ }^{\circ} \mathrm{C}$ for 3-4 h.

\section{Results and discussion}

In conventional heating, reactants are slowly activated by a conventional external heat source. Heat is driven into the substance, firstly passing through the walls of the vessel in order to reach the solvent and the reactants. On the other hand, microwave penetrates inside the material and heat is generated through direct microwave-material interaction. Since microwaves couple directly with the molecules of the entire reaction mixture, it leads to a rapid rise in the temperature. In this case, only the reaction vessel contents are
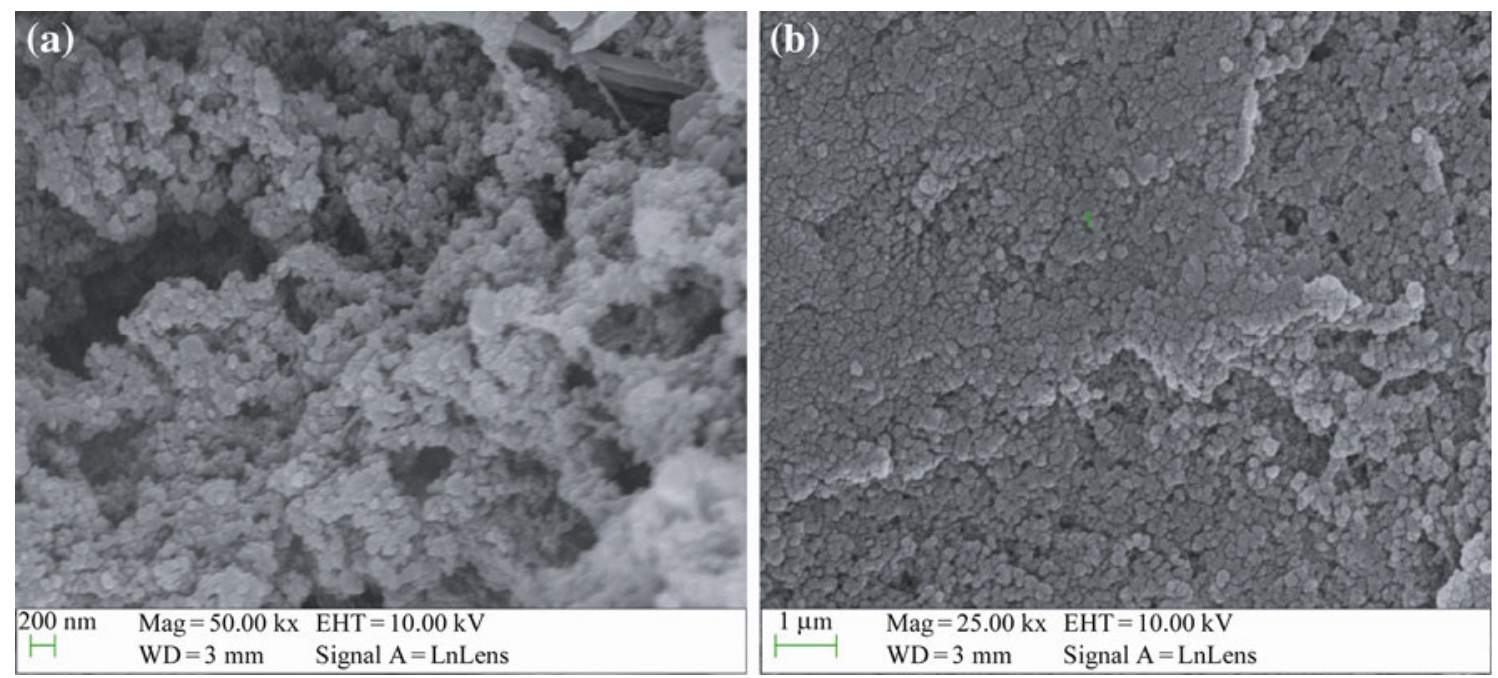

Fig. 1 SEM images of AlNP using tea leaves extract 


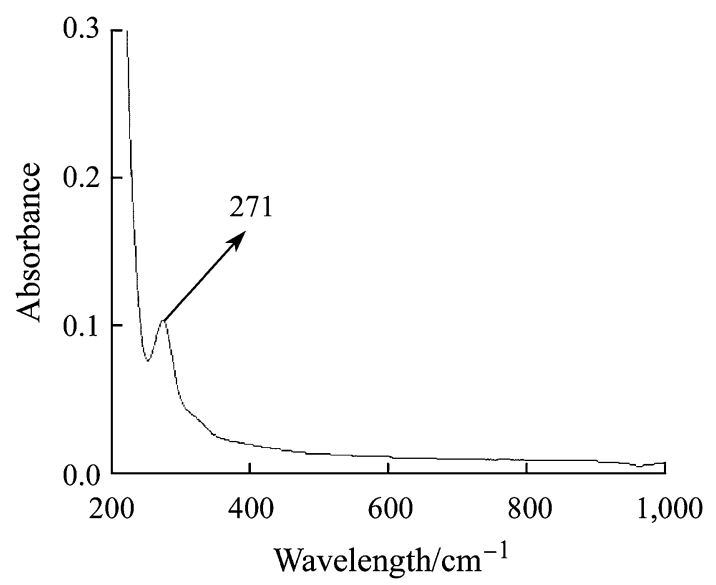

Fig. 2 UV-Vis of AlNP using tea leaves extract

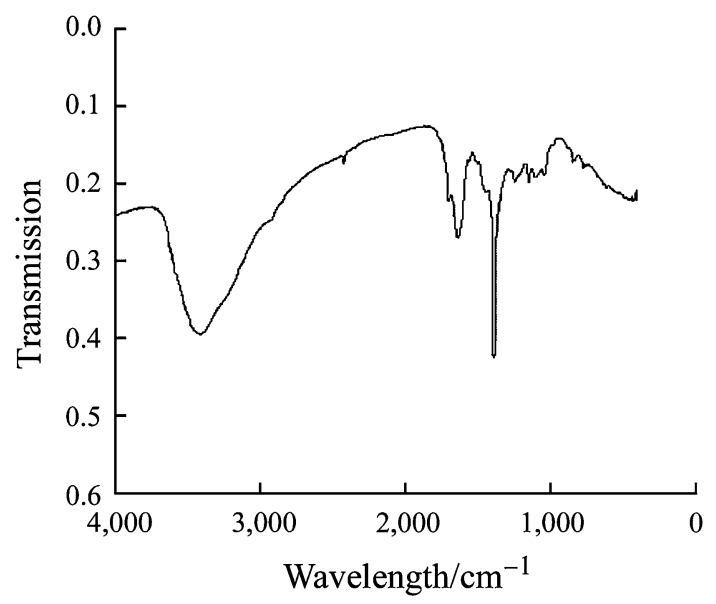

Fig. 3 FTIR of AINP using tea leaves extract
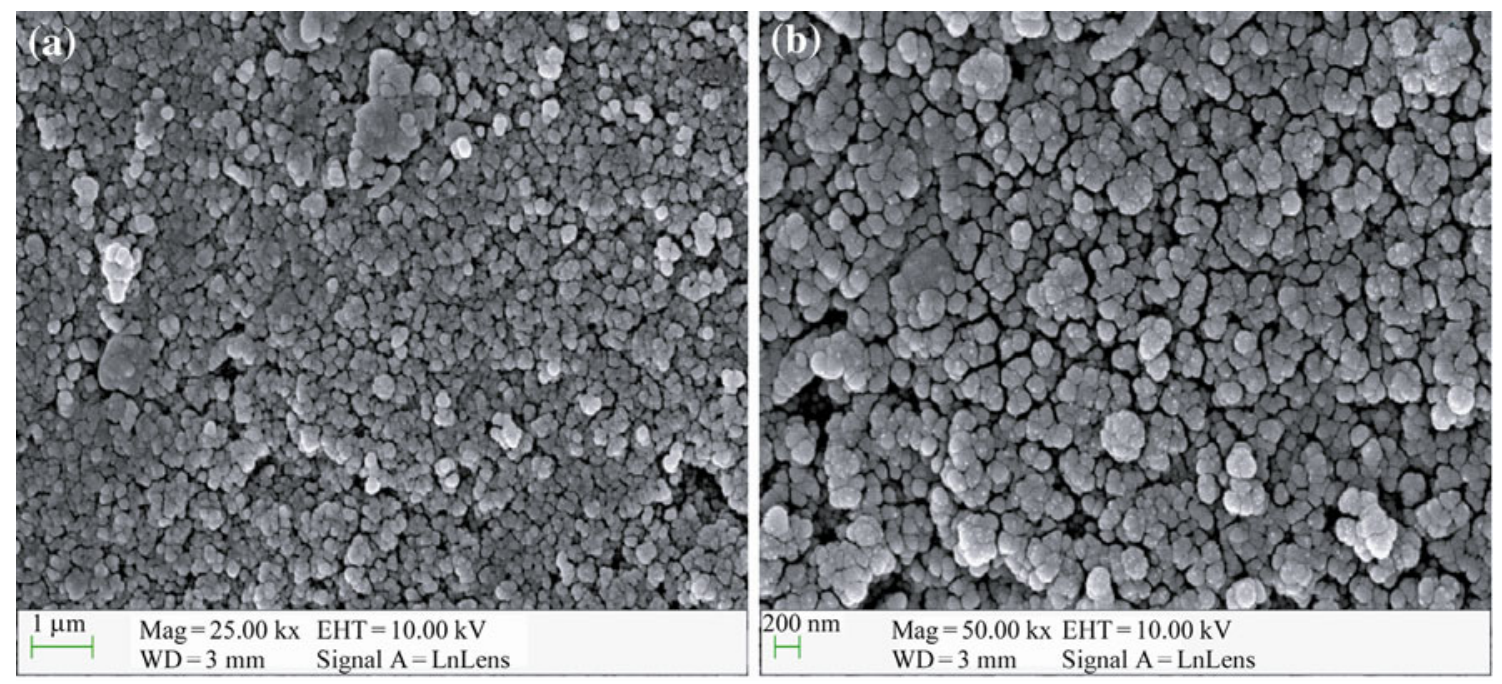

heated and not the vessel itself, hence better homogeneity and selective heating of polar molecules should be achieved. Therefore, for microwave heating, the substance must possess a dipole moment [20].

The formation of AlNP by the supernatant of the tea, coffee and triphala extracts were initially observed through colour change. The SEM of AINP using tea extract clearly indicates the formation of spherical AlNP with the size ranging between $50 \mathrm{~nm}$ and $100 \mathrm{~nm}$ as shown in the Fig. 1a, b. The formation of nanoparticles by the supernatant of the tea extract was monitored by periodic sampling of aliquots $(1 \mathrm{ml})$ and subsequently measuring $\mathrm{UV}-\mathrm{V}$ is spectra of these solutions between $200 \mathrm{~nm}$ and 1,000 $\mathrm{nm}$. Distilled water was used to adjust the baseline. Thus, the UV-Vis spectrum of AlNP from tea extract shows the peak at $271 \mathrm{~nm}$ (see Fig. 2). Figure 3 shows the Fourier transform infrared spectroscopy (FTIR) peaks of bio-functionalized AlNP at 1,630, 1,383, and $1,090 \mathrm{~cm}^{-1}$. The peak at $1,090 \mathrm{~cm}^{-1}$ indicates the presence of $\mathrm{C}-\mathrm{N}$ stretching frequency, whereas the geminal methyl group at $1,383 \mathrm{~cm}^{-1}$ and peak at $1,630 \mathrm{~cm}^{-1}$ represent the unreacted ketone group, suggesting the presence of flavonones adsorbed on the surface of AlNP. This suggests that influence of water soluble organic moieties of tea is responsible for the synthesis of AlNP and surface modification. The FTIR spectrum also shows a broad absorption band at $3,440 \mathrm{~cm}^{-1}$ is mainly ascribed to $\mathrm{OH}$ - groups on the surface of the AlNP nanostructure. The peak at $523 \mathrm{~cm}^{-1}$ indicates the formation of the AlNP nanostructures.

Figure $4 a, b$ show the SEM images of AlNP using coffee extract, and the results indicate the formation of spherical shaped nanoparticles below the size of $100 \mathrm{~nm}$. Absorption spectrum of AlNP has an absorbance peak at 275 nm (see Fig. 5). The FTIR spectrum shown in Fig. 6

Fig. 4 SEM images of AlNP using coffee extract 


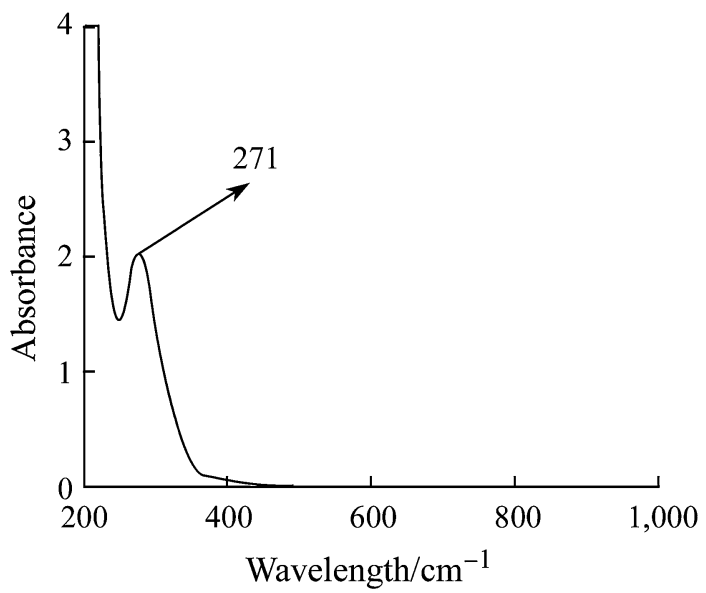

Fig. 5 UV-Vis of AlNP using coffee extract

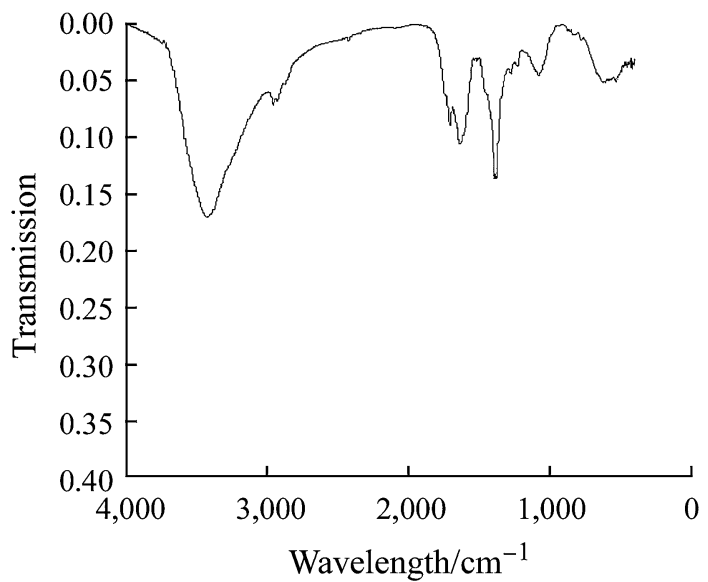

Fig. 6 FTIR of AlNP using coffee extract

again confirms the formation of AlNP in presence of coffee extract. The SEM images shown in Fig. 7a, b show the oval shaped structures of AlNP in 200-400 nm size, when triphala extract is used as reducing agent. The UV-Visible spectrum of AlNP from triphala extract shows the peak at
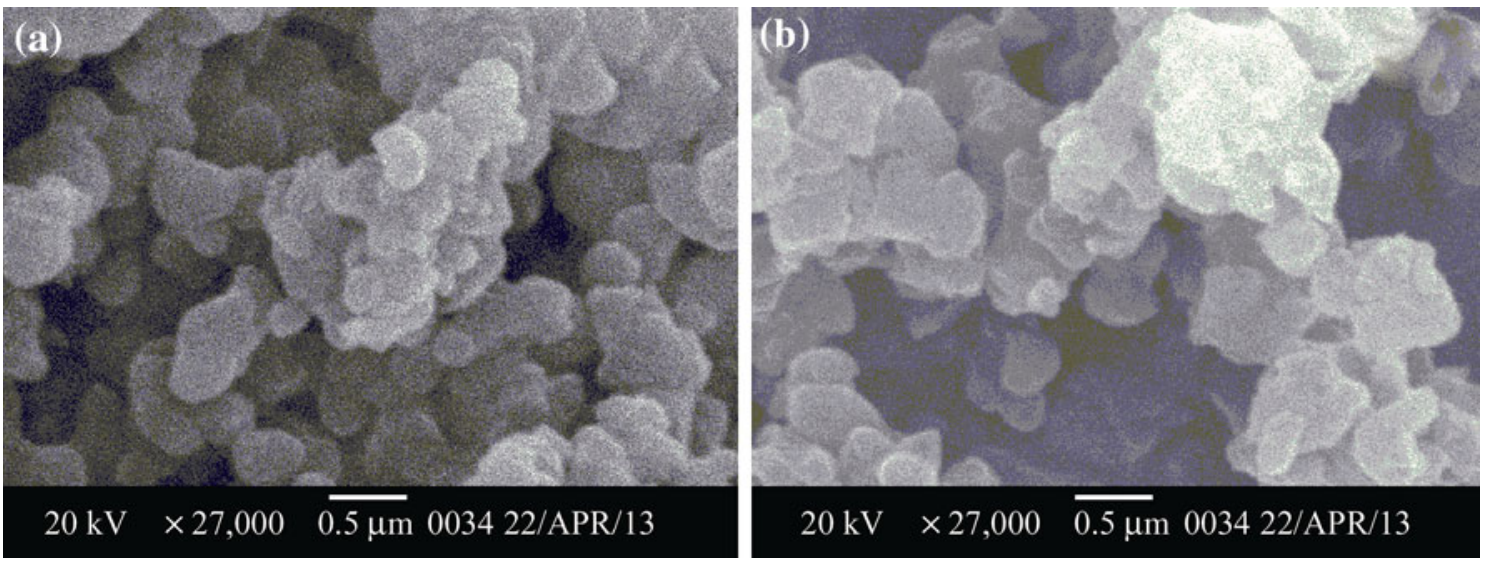

$273 \mathrm{~nm}$ (see Fig. 8). FTIR is shown in Fig. 9, showing peaks at $1,633,1,380$ and $1,080 \mathrm{~cm}^{-1}$. This indicates the secretion of some water soluble organic components, which might contribute for the reduction of aluminium nitrate into AlNP.

Actually, the polyphenols in tea, coffee and triphala are responsible for the chemical reduction of aluminium nitrate. In tea, the polyphenol content is less than coffee and triphala. NMR and FTIR study show that it mainly contains gallic acid, which might have increased the reaction rate, and therefore it took less time (2-3 $\mathrm{min}$ ) for the reduction of aluminium nitrate. The reaction does happen in absence of microwave heating, but it takes almost $7-8 \mathrm{~h}$ at room temperature. The particles shape are found to be uneven, much larger than those of microwave method. It can be inferred that the agglomeration of particles is prevented by the polyphenols content and thus confers stability of AlNP in the aqueous medium. This gives strong evidence for the polyphenols involvement in

Fig. 7 SEM images of AlNP using triphala extract 


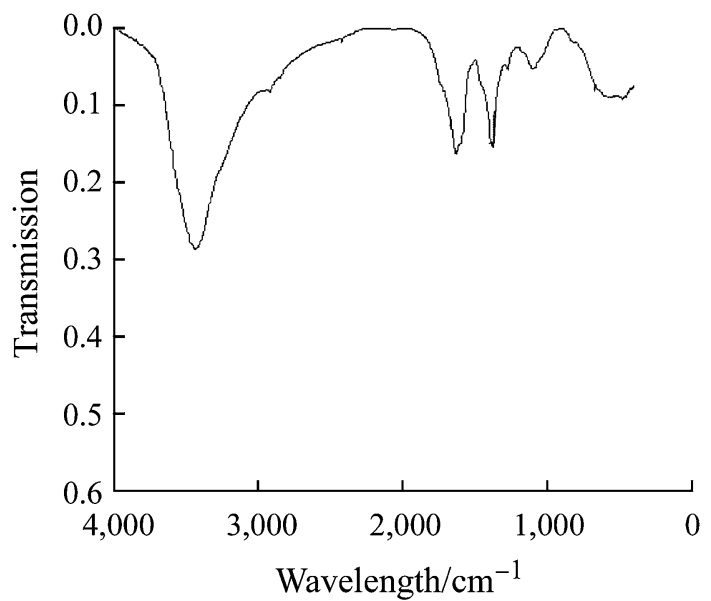

Fig. 9 FTIR of AlNP using triphala extract

the rapid biosynthesis and for the stability of metallic nanoparticles in the aqueous medium [21].

\section{Conclusions}

In this paper, microwave-assisted green chemistry has been used for the synthesis of AlNP. A facile approach has been reported using tea, coffee and triphala extracts, acting as reducing agents for the synthesis of AlNP of well-defined dimensions in bulk amount. This eliminates the need of toxic chemicals for the synthesis of nanoparticles. The formation of AlNP with the microwave-assistance using these plant extracts has proved to be very faster than any other method. These nanoparticles which are synthesized using green chemistry can be used for a variety of applications in the future. As triphala extract has medicinal importance, the results described here will also be useful for its biomedical applications.

Acknowledgments Authors express their gratitude to the Director, National Institute of Technology, Agartala to allow for publishing the results. Financial assistance from CPRI, Bangalore is greatly acknowledged.

\section{References}

1. Dillon AC, Mahan AH, Deshpande R et al (2008) Metal oxide nano-particles forimproved electrochromic and lithium-ion battery technologies. Thin Solid Films 516:794-797

2. Lach R, Haberko K, Bu'cko MM et al (2011) Ceramic matrix composites in the alumina/5-30 vol\% YAG system. J Eur Ceram Soc 31:1889-1895
3. Lee CL, Wan CC, Wang YY et al (2001) Synthesis of silver nanoparticles using hydroxyl functionalized ionic liquids and their antimicrobial activity. Adv Funct Mater 11:344-347

4. Wei S, Wang Q, Zhu J et al (2006) Alumina nanoparticles related composites application: surface functionalized alumina nanoparticle filled polymeric nanocomposite with enhanced mechanical properties. J Mater Chem 16:2800-2808

5. Guoa Z, Kumar CSSR, Henry LL et al (2005) Displacement synthesis of $\mathrm{Cu}$ shells surrounding Co nanoparticles. J Electrochem Soc 152(1):D1-D5

6. Capek I (2004) Preparation of metal nanoparticles in water-in-oil (w/o) microemulsions. Adv Colloid Interface Sci 110:49-74

7. Yin BS, Ma HY, Wang SY et al (2003) Electrochemical synthesis of silver nanoparticles under protection of poly $(\mathrm{N}$-vinylpyrrolidone). J Phys Chem B 107:8898-8904

8. Murphy CJ, San TK, Gole AM et al (2005) Anisotropic metal nanoparticles: synthesis, assembly, and optical applications. J Phys Chem B 109:13857-13870

9. Kumar SS, Manav S, Mitali S et al (2010) Carbon nanocubes and nanobricks from pyrolysis of rice. J Nanosci Nanotechnol 10:4064-4067

10. Mitali S, Soma D (2013) Electrochemical studies of carbon nanotube obtained from coconut oil as non enzymatic glucose biosensor. Adv Sci Eng Med 5:645-648

11. Soma D, Mitali S (2012) Preparation of carbon nanosphere from bamboo and its use in water purification. Curr Trends Technol Sci 2:174-177

12. Chandran SP, Chaudhary M, Pasricha R et al (2006) Synthesis of gold nanotriangles and silver nanoparticles using aloe vera plant extract. Biotechnol Prog 22:577-583

13. Moulton MC, Braydich-Stolle LK, Nadagouda MN et al (2010) Synthesis, characterization and biocompatibility green synthesized silver nanoparticles using tea polyphenols. Nanoscale 2:763-770

14. Nadagouda MN, Varma RS (2006) Green and controlled Synthesis of gold and platinum nanomaterials using vitamin B2: density-assisted self-assembly of nanospheres, wires and rods. Green Chem 8:516-518

15. Xin Y, Li Q, Huixuan W et al (2010) Green synthesis of palladium nanoparticles using broth of Cinnamomum camphora leaf. J Nanopart Res 12:1589-1598

16. Luki'c I, Krsti'c J, Jovanovi'c D et al (2009) Alumina/silica supported $\mathrm{K}_{2} \mathrm{CO}_{3}$ as a catalyst for biodiesel synthesis from sunflower oil. Bioresour Technol 100:4690-4696

17. Keyvani A, Saremi M, HeydarzadehSohi M (2010) Microstructural stability of zirconia-alumina composite coatingsduring hot corrosion test at $1050{ }^{\circ} \mathrm{C}$. J Alloys Compd 506:103-108

18. Liu IL, Shen $\mathrm{P}$, Chen SY (2010) $\mathrm{H}^{+}$- and $\mathrm{Al}^{2+}$-codoped $\mathrm{Al}_{2} \mathrm{O}_{3}$ nanoparticles with spinel- type related structures by pulsed laser ablation in water. J Phys Chem C 114:7751-7757

19. Monica D, Soma D, Mitali S (2013) Effect of reducing agents on the structure of zinc oxide under microwave irradiation. Adv Manuf 1:183-186

20. Surati MA, Smita J, Desai KR (2012) A brief review: microwave assisted organic reaction. Arch Appl Sci Res 4(2):645-661

21. Kamat PV (2002) Photophysical, photochemical and photocatalytic aspects of metal nanoparticles. J Phys Chem B 106(32): 7729-7744 